\title{
Double $K$-Vacancy Production by X-Ray Photoionization
}

\author{
S. H. Southworth*, R. W. Dunford*, E. P. Kanter*, B. Krässig *, L. Young*, \\ G. B. Armen ${ }^{\dagger}$, J. C. Levin ${ }^{\dagger}$, M. H. Chen ${ }^{* *}$ and D. L. Ederer ${ }^{\ddagger}$ \\ *Argonne National Laboratory, Argonne, IL 60439, USA \\ ${ }^{\dagger}$ University of Tennessee, Knoxville, TN 37996, USA \\ ${ }^{*}$ Lawrence Livermore National Laboratory, Livermore, CA 94550, USA \\ ${ }^{\ddagger}$ Tulane University, New Orleans, LA 70118, USA
}

\begin{abstract}
.
We have studied double $K$-shell photoionization of Ne and Mo $(\mathrm{Z}=10$ and 42) at the Advanced Photon Source. Double $K$-vacancy production in Ne was observed by recording the $K K-K L L$ Auger hypersatellite spectrum. Comparison is made with calculations using the multiconfiguration Dirac-Fock method. For Mo, double $K$-vacancy production was observed by recording the $K \alpha, \beta$ fluorescence hypersatellite and satellite $\mathrm{x}$ rays in coincidence. From the intensities of the Auger or x-ray hypersatellites relative to diagram lines, the probabilities for double $K$-vacancy production relative to single $K$-vacancies were determined. These results, along with reported measurements on other atoms, are compared with Z-scaling calculations of the high-energy limits of the doubleto-single $K$-shell photoionization ratio.
\end{abstract}

\section{Introduction}

Double photoionization of He has been extensively studied because it is sensitive to electron correlation in the simplest case of a two-electron system [1]. Calculation of the double-to-single photoionization ratio $\mathrm{R}$ as a function of photon energy provides a testing ground for the development of theories that incorporate electron correlation in the ground state and in the final continuum states $[2,3]$. Among several theoretical approaches [3], the many-body perturbation theory (MBPT) [4] provides a description of the double-photoionization process in terms of three first-order amplitudes in the electron-electron interaction (see [2,3] for discussions of the MBPT). The three lowestorder MBPT diagrams are referred to as GSC (ground state correlation), SO (shake off), and TS1 (two-step one). The GSC accounts for radial and angular correlation between the two equivalent $1 \mathrm{~s}$ electrons in the ground state (see, e.g., [5]). SO accounts for relaxation in the final ionic state upon ejection of one of the electrons into the continuum. The TS1 is also a final state interaction and describes photoemission of one electron that knocks out the second electron by binary collision.

The MBPT amplitudes are added coherently, so describing the doublephotoionization process in terms of their relative importance should be done cautiously. The amplitudes are also gauge dependent, i.e., they depend on the form of the dipole operator used (length, velocity, or acceleration), a topic that has been discussed in the theoretical literature $([2,3,6])$. Nevertheless, it is informative to consider the 
energy dependencies of the three amplitudes in the acceleration form of the dipole operator [4] which gives better agreement with the measurements of [1] than the length and velocity results. All three amplitudes increase strongly from threshold, but the GSC and SO amplitudes level out asymptotically, while the TS1 amplitude passes through a maximum and decreases monotonically to high energy. The picture that emerges is that $\mathrm{R}$ increases from threshold to a maximum, then falls off slowly to an asymptotic ratio determined by the GSC and SO terms. This general picture is supported by Z-scaling calculations of double $K$-shell photoionization of two-electron systems [7].

High-energy-limit calculations [8, 9] are interesting and useful, because they are considered to be asymptotically exact and provide reference values for experiments on atoms other than He. In the high energy limit, final-state correlation is negligible and only the ground-state correlation needs to be accurately treated. Very accurate $1 s^{2}$ ground state wavefunctions can be constructed for $\mathrm{He}$ and He-like ions [8, 9]. The results of those calculations provide reference values for experiments that measure $R$ of many-electron targets under the assumption that interaction with outer-shell electrons is negligible. However, scaling shows a $Z^{2}$ dependence on the energy required to reach the asymptotic region [7] and may be difficult to achieve for higher- $Z$ atoms. Good agreement is obtained between theory and the high-energy measurement $(5.4-9.1 \mathrm{keV})$ on He using the ion-recoil momentum imaging method to distinguish photoabsorption from Compton scattering [10]. The experiments on higher-Z atoms described here and in other publications $[1,11,12,13]$ were measured using photon energies close to the expected maxima of $\mathrm{R}$ [7]. Calculations that treat energy-dependent correlation interactions for this ratio are lacking for atoms other than He. The experiments may motivate more detailed calculations on higher- $Z$ atoms that may require treatment of relativistic interactions as well as electron correlation.

\section{$\mathrm{Ne}[\mathrm{KK}]$ at $5 \mathrm{keV}$}

The threshold for double- $K$ ionization of $\mathrm{Ne}$ is $1863 \mathrm{eV}$ [14] compared with 870.21 $\mathrm{eV}$ for single- $K$ ionization [5]. Ne $K$ vacancies decay predominantly by emission of $K$-LL Auger electrons rather than by fluorescence [15]. The energies and relative intensities of diagram Auger lines and the satellites resulting from additional excitation or ionization of the $L$ shell have been determined experimentally $[16,17]$. Using the multiconfiguration Dirac-Fock method, Chen [18] calculated the energies and transition rates of the $K K$-KLL Auger hypersatellite spectrum. The hypersatellites were used to determine $\mathrm{R}$ for $\mathrm{Ne}$ by electron impact [14], and the same method was used here. For the present experiment, double- $K$ vacancies were produced in Ne by photoionization with a $5 \mathrm{keV}$ photon beam at Argonne's Advanced Photon Source (APS), and the resulting $K$ $L L$ and $K K-K L L$ Auger spectra were recorded with a cylindrical-mirror electron analyzer (CMA). The CMA was positioned with its symmetry axis in the plane perpendicular to the $\mathrm{X}$-ray beam and at the magic angle $\left(54.7^{\circ}\right)$ with respect to the linear-polarization direction to eliminate the dependence of measured intensities on angular anisotropies. The electron spectra were corrected for energy variations of the collection efficiency of the CMA and for variations of the $\mathrm{x}$-ray beam intensity. The spectrum in Fig. 1 shows the 
$K-L_{2,3} L_{2,3}{ }^{1} D$ diagram line at $804.5 \mathrm{eV}$ and the $K K-K L_{2,3} L_{2,3}{ }^{2} D$ hypersatellite line at $870.5 \mathrm{eV}$. The structure between $835-855 \mathrm{eV}$ is due to the $K K-K L_{1} L_{2,3}{ }^{2} P^{(+)}$hypersatellite and satellites from the decay of initial states such as [1s2p]np and [1s2s]ns, i.e., shakeup states observed in the 1s photoelectron spectrum [16, 19].

The area of the $K-L_{2,3} L_{2,3}{ }^{1} D$ diagram peak is proportional to the number of single $K$-vacancies produced times the branching ratio into that particular final state. The branching ratio was deduced from experimental results $[15,17]$. Similarly, the area of the $K K-K L_{2,3} L_{2.3}{ }^{2} D$ peak is proportional to the number of double $K$-vacancies produced times its branching ratio. The branching ratio was determined from the calculated Auger rates [18] and estimates of the probabilities of fluorescence and multiple-Auger final states. From this analysis, the ratio $\mathrm{R}$ for $\mathrm{Ne}$ at $5 \mathrm{keV}$ was determined and is plotted in Fig. 3 below with results for other atoms. We stress that the result reported here is preliminary due to the need to fully account for weak Auger satellites from other initial states.

\section{Mo[KK] at $50 \mathrm{keV}$}

A full report on our measurement of $\mathrm{R}$ for $\mathrm{Mo}$ at $50 \mathrm{keV}$ is given in [13]. The threshold for double- $K$ ionization is $40.654 \mathrm{keV}$ compared with $20 \mathrm{keV}$ for single- $K$ ionization. Mo $K$ vacancies decay predominantly by x-ray fluorescence, and double $K$ vacancies emit a $K K-K L$ hypersatellite $\mathrm{x}$ ray shifted up in energy from the $K \alpha, \beta$ diagram lines followed by a $K L-L^{2}$ satellite. Our experiment at the APS used x-ray beams of 40.2 and $50 \mathrm{keV}$ passing through a thin Mo target. Two $\mathrm{Si}(\mathrm{Li})$ detectors faced each other with the target inbetween and were normal to the beam and parallel to the polarization direction to minimize detection of scattered $\mathrm{x}$ rays. Coincidence electronics recorded the $\mathrm{x}$-ray energies deposited in each detector and their time difference. The energy spectra recorded in each detector for coincidences with a $K \alpha, \beta$ x-ray (encompassing also the $K L-L^{2}$ satellite) are plotted in Fig. 2. The real coincidences show weak shoulders on the high-energy sides of the diagram lines due to the hypersatellites. These shoulders were absent in the coincidence spectra recorded at the subthreshold energy of $40.2 \mathrm{keV}$. The ratio $\mathrm{R}$ was determined from the relative intensities of hypersatellites and diagram transitions after accounting for solid angles, detection efficiencies, and fluorescence yields [13].

Our results for the double-to-single $K$-shell photoionization ratio R for Ne at $5 \mathrm{keV}$ and $\mathrm{Mo}$ at $50 \mathrm{keV}$ are plotted in Fig. 3 along with results for other atoms [1, 10, 11, 12]. For reference, the calculated asymptotic Z-scaling law of [8] is also shown. Except for the high-energy measurement on $\mathrm{He}$ [10], the measured ratios are significantly higher than the asymptotic limit, as expected. With the exception of [10], the measurements were made at photon energies within the broad maximum of $\mathrm{R}$ predicted by Z-scaling calculations [7]. The solid line through the measurements is a power-law fit with the form $1 / Z^{1.61}$ [13]. This dependence on $\mathrm{Z}$ for values of $\mathrm{R}$ measured near their maxima is somewhat weaker than that calculated for the asymptotic limits [8]. 


\section{ACKNOWLEDGMENTS}

We are grateful to the staff of the Basic Energy Sciences Synchrotron Radiation Center at the Advanced Photon Source for their assistance in performing the experiments. The Argonne group is supported by the Chemical Sciences, Geosciences, and Biosciences Division of the Office of Basic Energy Sciences, Office of Science, U.S. Department of Energy, under Contract No. W-31-109-Eng-38. Use of the Advanced Photon Source is supported by the U. S. Department of Energy, Basic Energy Sciences, Office of Science, under Contract No. W-31-109-Eng-38.

\section{REFERENCES}

1. Samson, J. A. R., Stolte, W. C., He, Z.-X., Cutler, J. N., Lu, Y., and Bartlett, R. J., Phys. Rev. A, 57, 1906 (1998).

2. McGuire, J. H., Berrah, N., Bartlett, R. J., Samson, J. A. R., Tanis, J. A., Cocke, C. L., and Schlachter, A. S., J. Phys. B, 28, 913 (1995).

3. Sadeghpour, H. R., Can J. Phys., 74, 727 (1996).

4. Hino, K., Ishihara, T., Shimizu, F., Toshima, N., and McGuire, J. H., Phys. Rev. A, 48, 1271 (1993).

5. Schmidt, V., Electron Spectrometry of Atoms using Synchrotron Radiation, Cambridge University, Cambridge, 1997.

6. Forrey, R. C., Yan, Z.-C., Sadeghpour, H. R., and Dalgarno, A., Phys. Rev. Lett., 78, 3662 (1997).

7. Kornberg, M. A., and Miraglia, J. E., Phys. Rev. A, 49, 5120 (1994).

8. Forrey, R. C., Sadeghpour, H. R., Baker, J. D., MorganIII, J. D., and Dalgarno, A., Phys. Rev. A, 51, 2112 (1995).

9. Krivec, R., Amusia, M. Y., and Mandelzweig, V. B., Phys. Rev. A, 63, 052708 (2001).

10. Spielberger, L., Jagutzki, O., Dörner, R., Ullrich, J., Meyer, U., Mergel, V., Unverzagt, M., Damrau, M., Vogt, T., Ali, I., Khayyat, K., Bahr, D., Schmidt, H. G., Frahm, R., and Schmidt-Böcking, H., Phys. Rev. Lett., 74, 4615 (1995).

11. Ahopelto, J., Rantavuori, E., and Keski-Rahkonen, O., Phys. Scr., 20, 71 (1979).

12. Diamant, R., Huotari, S., Hämäläinen, K., Kao, C. C., and Deutsch, M., Phys. Rev. A, 62, 052519 (2000).

13. Kanter, E. P., Dunford, R. W., Krässig, B., and Southworth, S. H., Phys. Rev. Lett., 83, 508 (1999).

14. Pelicon, P., Čadež, I., Žitnik, M., Ž. Šmit, Dolenc, S., Mühleisen, A., and Hall, R. I., Phys. Rev. A, 62, 022704 (2000).

15. Kanngießer, B., Jainz, M., Brünken, S., Benten, W., Gerth, C., Godehusen, K., Tiedtke, K., van Kampen, P., Tutay, A., Zimmermann, P., Demekhin, V. F., and Kochur, A. G., Phys. Rev. A, 62, 014702 (2000).

16. Krause, M. O., Carlson, T. A., and Moddeman, W. E., J. Phys. (Paris), 32, C4-139 (1971).

17. Albiez, A., Thoma, M., Weber, W., and Mehlhorn, W., Z. Phys. D, 16, 97 (1990).

18. Chen, M. H., Phys. Rev. A, 44, 239 (1991).

19. Svensson, S., Eriksson, B., Mårtensson, N., Wendin, G., and Gelius, U., J. Electron Spectrosc., 47, 327 (1988). 


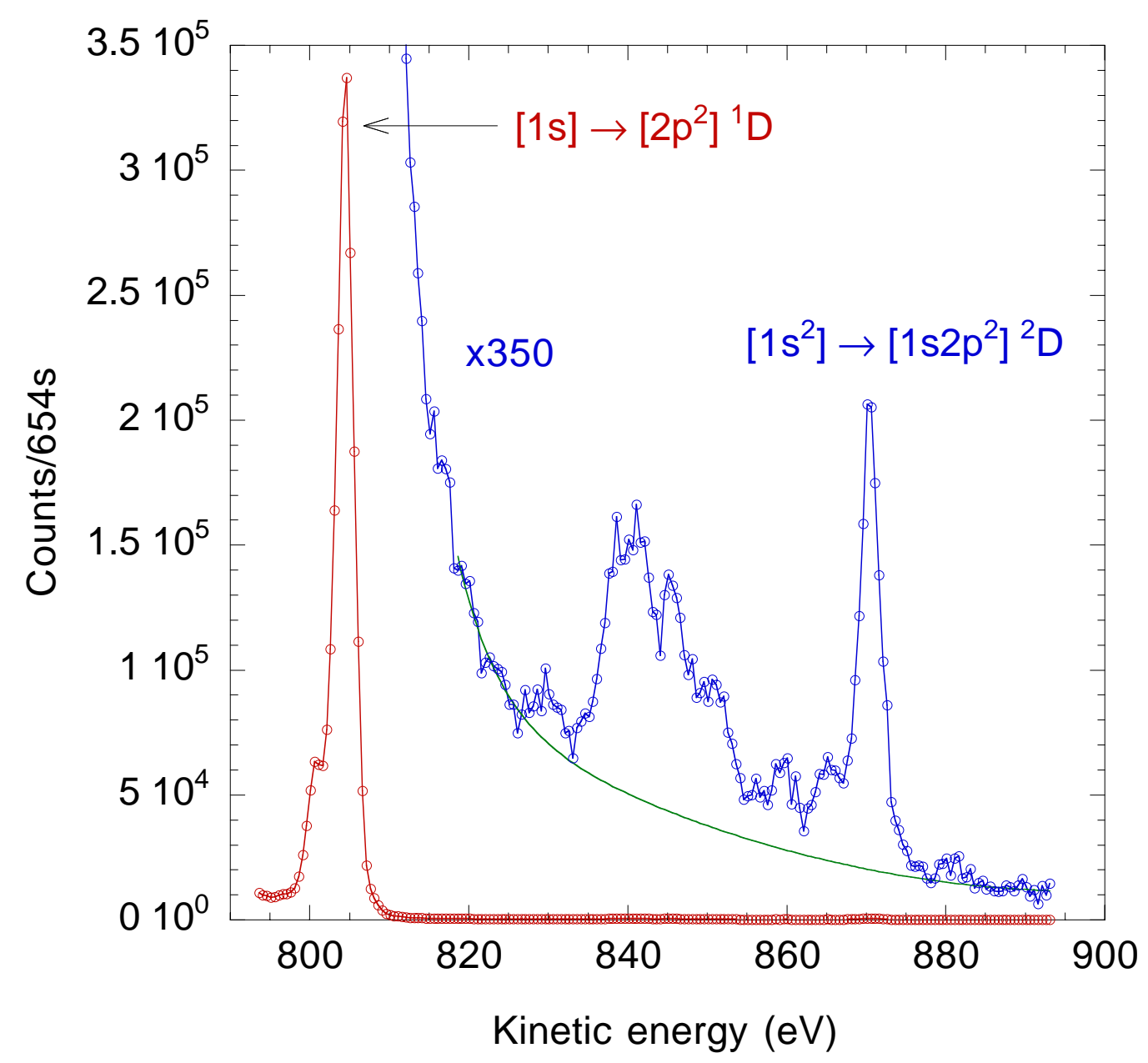

FIGURE 1. Auger electron spectrum of Ne excited by absorption of $5 \mathrm{keV}$ x rays. The $K-L_{2,3} L_{2,3}{ }^{1} D$ diagram line at $804.5 \mathrm{eV}$ and the $K K-K L_{2,3} L_{2,3}{ }^{2} D$ hypersatellite line at $870.5 \mathrm{eV}$ are indicated. The structure between $835-855 \mathrm{eV}$ is discussed in the text. 


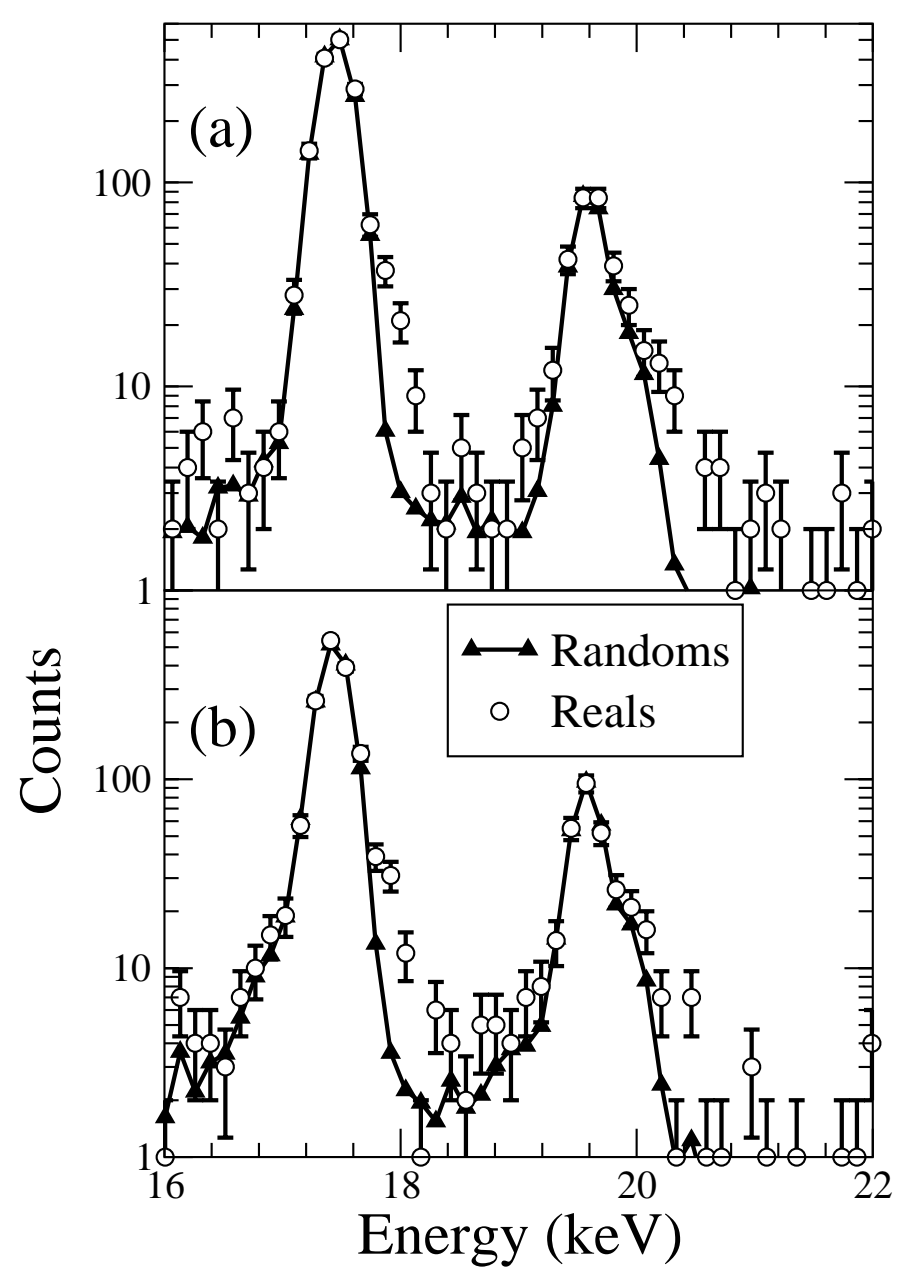

FIGURE 2. $\mathrm{X}$ ray/x ray fluorescence coincidence spectra from Mo excited by absorption of $50 \mathrm{keV}$ $\mathrm{x}$ rays. The energy spectra in each detector are shown for coincidences with $K \alpha, \beta \mathrm{x}$ rays in the other detector. The spectrum for detector " 1 " is in (a) while that for " 2 " is in (b). The data plotted as open circles are for real coincidences while the filled triangles with solid curve are random coincidences. 


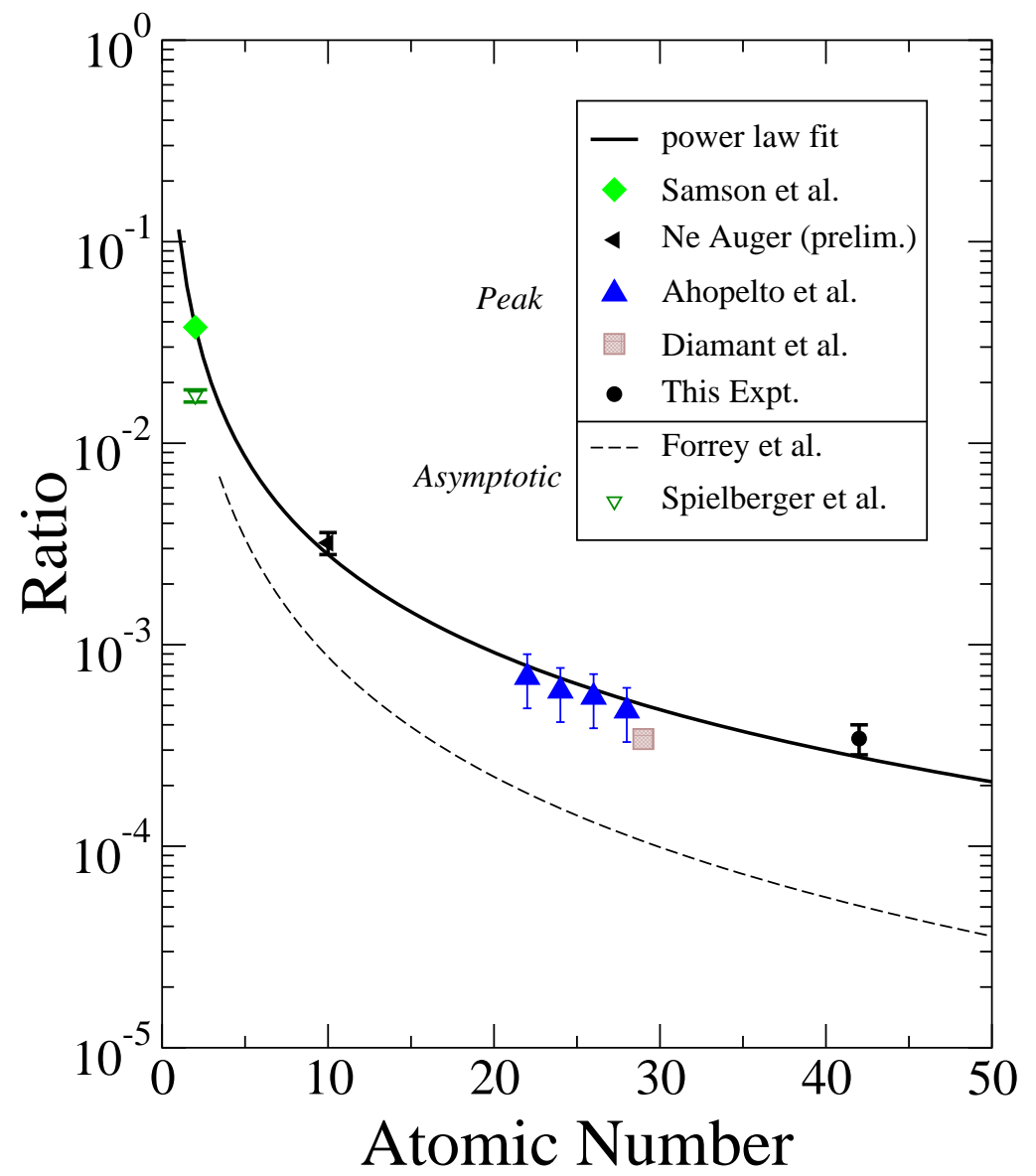

FIGURE 3. The ratio of double-to-single $K$-shell photoionization as a function of atomic number. Measured results are for $\mathrm{He}$ at $200 \mathrm{eV}$ [1] (diamond), $\mathrm{He}$ at 5.4-9.1 keV [10] (open triangle), $\mathrm{Ne}$ (this work; left triangle), $\mathrm{Ti}, \mathrm{Cr}, \mathrm{Fe}$, and $\mathrm{Ni}$ [11] (up triangle), $\mathrm{Cu}$ [12] (square), and $\mathrm{Mo}$ [13] (solid circle). The solid curve is a power law fit to the measurements. The dashed curve shows the Z-scaling law calculated for He-like ions in the high-energy limit [8]. 